\title{
Photographic Investigations of Jet Disintegration in Airblast Sprays
}

\author{
S.N. Sridhara ${ }^{1}$ and B.N. Raghunandan ${ }^{2}$ \\ ${ }^{I}$ Professor, Department of Mechanical and Automotive Engineering, MS Ramaiah School of Advanced Studies, New \\ BEL Road, MSR Nagar, Bangalore-560 054, INDIA \\ ${ }^{2}$ Professor, Dept. of Aerospace Engineering, Indian Institute of Science, Bangalore-560012, INDIA
}

Email: sridharasn1964@gmail.com,sns@msrsas.org

(Received January 22, 2009; accepted June 23, 2009)

\begin{abstract}
In this paper a comprehensive review of flow visualization techniques used for the analysis of spray characteristics is presented and an experimental demonstration of different lighting techniques has been made. Still photographs of sprays from an airblast atomizer and a swirl atomizer are taken under backlighting, slit lighting, incident lighting (front flash) and a new method of capturing scattered light image at 1200 to the incident light is introduced in the present work. Photographs taken by these methods are shown to be useful for deriving useful observations such as jet breakup phenomenon, the internal details and the external appearance of the spray. It is demonstrated that the information obtainable from each technique is different and that a combination of different techniques may be necessary for proper diagnosis. The article also includes a discussion on the jet breakup phenomenon as observed in the photographs and in addition a new concept of shear formation of smaller droplets from the surface of a liquid jet by the co-flowing air stream in an airblast atomizer has been proposed.
\end{abstract}

Keywords: Airblast atomizer, jet breakup, visualization, jet instability

\section{INTRODUCTION}

Many investigators have employed flow visualization in spray applications for qualitative and quantitative analysis. The drop sizing and drops dispersion ' $\cdots$ (Jones 1977; Hay et al. 1998), breakup phenomenon, breakup length (Dombrowski and Fraser 1954; Fraser et al. (1963a, 1963b, 1963c); Chigier and Reitz 1995; Eroglu and Chigier 1991), and external characteristics of the sprays such as cone angle (Shivakumar and Raghunandan 1996) etc, have been analyzed with varying degrees of success. Lighting sources such as mercury vapour lamps, electrical sparks, flash light sources, strobe light, halogen lamps and pulsed laser sheets are commonly employed for the purpose. Flash light sources of approximately one millisecond duration and laser pulses with duration as small as nanoseconds with still camera or halogen lamp with high-speed cine camera are used for 'freezing' the spray.

Commonly reported lighting methods in spray diagnostics involve backlighting (Fraser et al. (1963a, 1963b, 1963c); Chigier and Reitz 1995; Eroglu et al. 1991; Mayer 1994; Wu et al. 1995; Carvalho et al. 2002); Schlieren (Mayer and Tamura 1996), illuminating a particular section of spray by a sheet of light (Carvalho et al. 2002; Candel et al. 1998), and incident lighting technique (Dombrowski and Fraser 1954; Shedd and Pautsch 2005; Kufffererath et al.
1999). However, a detailed study of such lighting methods with special emphases to the exploration of different characteristics of spray is seldom illustrated in the literature.

An effort is made in the current study to fill this void in terms of a review of various lighting techniques and experimental demonstration of these techniques in the spray diagnosis. A new method of capturing the elastic scattered light at $120^{\circ}$ to the incident light has been introduced in the study, which is shown to be useful to capture the finer details of spray boundary.

\section{REVIEW OF LITERATURE WORK}

Backlighting method is basically a shadowgraph method, in which the shadow of the object is recorded on the photographic film. In principle, shadow represents the second derivative of density variation in the field of observation (Liepmann and Roshko 1963). A large ratio of densities of fluids in a two-phase flow such as in spray field ensures a sharp and clear image of liquid mass with this technique. This method with the use of microflash finds extensive application in the analysis of jet breakup phenomenon due to its ability to freeze the liquid jet/sheet and droplets with a highresolution image of jet/sheet disintegration. Early works by Fraser et al. (1963a, 1963b, 1963c), and the recent studies by Eroglu and coworkers (1991), Farago 
and Chigier (1991) and Chigier and Reitz (1995) and Carvalho et al. (2002) are a few examples that have exploited this technique.

The image in shadowgraphy may be captured in two ways. In the first method, the camera lens is focused to the centerline of spray, so that the liquid droplets that are in focus would appear sharp and the rest with dull boundaries as may be observed in some of the publications (Eroglu et al. 1991; Farago and Chigier 1991; Chigier and Reitz 1995; Carvalho et al. 2002; Cavelere et al. 2003). This would enable one to subtract the effect of thick cloud of droplets in a dense and wide spray and highlight only the liquid core at the centre. Hay et al. (1998) also demonstrated the possibility of drop size measurement with this method coupled with image processing via CCD camera and microcomputer. In this work, an effective gradient in the image intensity is used to eliminate the drops that are out of focus. Perhaps this was the first effort in the quantitative analysis of image obtained in shadowgraphy.

In another method, the image is received onto a translucent screen and the camera lens is focused on this image from the other side of the screen (Carvalho and Heitor 1998). Due to enlarged image of droplets that are closer to the illuminating source and relatively smaller images of droplets that are closer to the screen, a nonrealistic disproportional image is obtained. Moreover, this technique fails to give much details of the inner structure of the spray especially in the case of dense and wide sprays due to the problem cited above.

In Schlieren system, a part of deflected light is intercepted before it reaches the viewing screen or photographic film. Thus, for a plane flow the increase or decrease of illumination at the screen is proportional to the density gradient in contrast to the shadowgraphy, in which this depends on the second derivative of density. Hence, Schlieren is commonly used wherever the quantitative evaluation of the image via density measurement is desired such as in gas flow and rarely used in two phase flow applications. However, Mayer and Tamura (1996) have successfully adopted this technique in the diagnosis of a burning spray where variation in gas phase density is encountered, and the authors were successful to derive well-defined photographs of burning liquid oxygen in the gaseous hydrogen medium. In a similar work by Tikkanen et al. (1997), the process of liquid flame spraying is analysed using Schlieren technique. In their study, Schlieren movie images were captured in a CCD camera at 30 frame per second and processed using a microprocessor. Authors claim to have studied the variation of flame structure, turbulence and stability of flame under various conditions using this technique. Katanada Hiroshi et al. (2005) used Schlieren technique for the study of supersonic flows in high velocity oxy-fuel thermal spray process and presented the intricate internal and external flow fields containing various types of shock and expansion waves. In all these studies (Mayer and Tamura 1996; Tikkanen et al. 1997; Katanada Hiroshi et al. 2005) the evaporating and combusting spray images were captured using Schlieren technique wherein the density difference in the flow field is minimal. The application of Schlieren technique to non-evaporating and non-combusting spray is seldom reported in the literature since the density gradients between the liquid and gas phase is large and shadowgraphy technique would give better images in such flow fields.

Illuminating a particular section of spray by a sheet of light (either pulsed or continuous) is a common technique used in the flow visualization of axisymmetric equidensity flows (Rehab et al. 1997). In this technique, the camera is positioned perpendicular to the sheet of light and the elastic-scattering signals are recorded on the photographic film. Since the elasticscattering signals emitted by the droplets perpendicular to the line of illumination is very weak, high power illumination and sensitive photographic film/sensor is essential to capture good quality photographs. This technique has also been applied to spray fields by many investigators (Shivakumar and Raghunandan 1996; Carvalho et al. 2002; Candel et al. 1998; Cavelere et al. 2003; Zeng et al. 1996; Soltani et al. 2005) with varied degrees of success. Sivakumar and Raghunandan (1996) used this technique in the analysis of dynamics of coaxial swirl jets in which the flow dynamics of inner liquid sheet normally gets obscured by the annular outer liquid sheet. The spray width in a swirl atomizer has been successfully measured using this technique by Zheng et al. (1996) and Soltani et al. (2005). The partial illumination of the spray by the sheet of light in this method may result in the partial absorption of light by the medium nearer to the illuminating edge and result in fading out of the image of the other half of the spray. This is especially true in case of dense sprays as can be observed in the work of Zheng et al. (1996). The tomographical studies of the liquid jet disintegration in high pressure air cross flow was carried out by Cavelere et al. (2003) in which a $0.5 \mathrm{~mm}$ thick Nd-YAG pulsed laser sheet was used to illuminate different cross sections of the spray. The images were captured in this study using a high resolution CCD camera placed at right angle to the light source. Since the Rayleigh scattered light intensities are weaker at $90^{\circ}$ to the source, elastically scattered light polarized components were selected by means of polariser sheet and then captured in the camera by these investigators Cavelere et al. (2003). The authors Cavelere et al. (2003) were successful in demonstrating the technique of obtaining tomographical views of the spray by using this technique.

In incident lighting (front illumination) technique (Dombrowski and Fraser 1954; Shedd and Pautsch 2005; Kufffererath et al. 1999), the camera and the illuminating source are placed parallel, hence the reflected light from the object is captured on the film. This technique is useful to record the external appearance of the spray, but fails to yield any inner details.

No previous work combines all these techniques and establishes the relative merit of each. This study is an effort towards filling this gap by adopting all these techniques except Schlieren for the non-combusting spray imaging and includes a critical analysis of the same. 


\section{EXPERIMENTAL SeTUP}

Sprays from two mechanistically different atomizers, namely airblast (Fig. 1) and swirl atomizers (Fig. 2) have been investigated in this study. Compressed air was supplied to the nozzle of airblast atomizer by four inlets at $150 \mathrm{~mm}$ from the nozzle faceplate. A flow straightener constructed by inserting drinking straws of inner diameter $4 \mathrm{~mm}$ and thickness $0.1 \mathrm{~mm}$ of total length of $50 \mathrm{~mm}$ in an annular tube of inner diameter 50 $\mathrm{mm}$ and outer diameter $80 \mathrm{~mm}$, was used to reduce flow asymmetry and residual swirling motion of air. Its performance was verified to give continuous supply of air without any fluctuation by traversing a Pitot tube across it in the assembled condition in the range of flow rates used in the measurement.

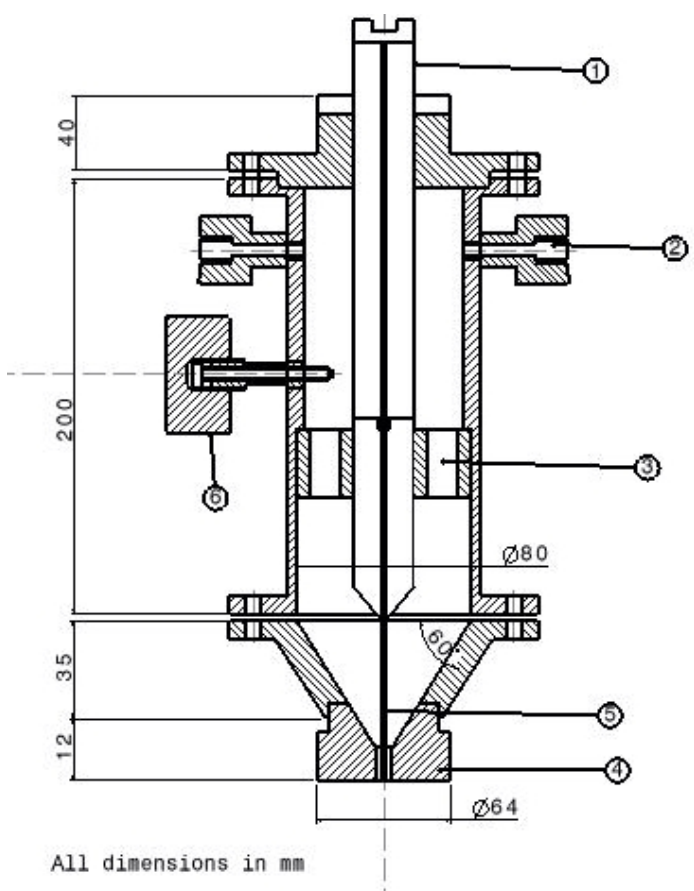

Fig. 1. Schematic of airblast atomizer used in the study. (Dimensions in $\mathrm{mm}$ ) (1) liquid tube (2) air inlet (4 No.) (3) Flow straightner (4) air nozzle end (ID: $6 \mathrm{~mm}$ ) (5) liquid nozzle end (OD: 3mm, ID: $1 \mathrm{~mm}$ ) (6) liquid tube centering pine assembly ( 3 No.)

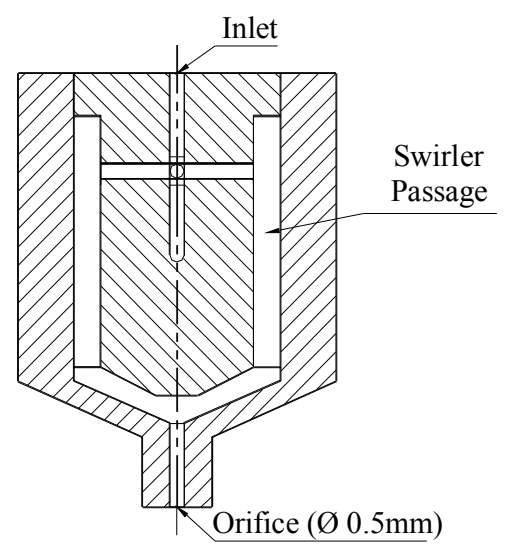

Fig. 2. Schematic of swirl atomizer used in the study.
The liquid tube was accurately positioned at the center of air nozzle with the help of three centering pins located 120 degree apart and the concentricity was verified using a travelling microscope before any measurement was taken. Both the air and water flow rates were precisely controlled through needle valves and accurately monitored through calibrated rotameters. The same water supply system was used for the study on swirl atomizer also. The schematic of experimental rig is shown in Fig. 3. The atomizers were operated at different mass flow rates of air and water in order to trace different stages of the spray formation of tulip like and hollow cone structures.

A Nikkon SLR camera with different close-up lenses and attachments was used with the flash unit discharging the required flashlight for a short duration. With several test cases it was established in the current study that a particle moving not more than $0.1 \mathrm{~mm}$ during the exposure time would result in an instant image in the frame of $2 \mathrm{X}$ magnification and any higher displacement results in the time integrated image. The minimum duration of flash required to capture the instant pictures of surface waves on the liquid jet and the moving drops detached from the liquid jet at near nozzle regions were established with prior knowledge of velocities involved. The velocity of water jet was maintained within $2 \mathrm{~m} / \mathrm{s}$ in the study and therefore the motion of surface waves on the liquid jet is expected to be of the same order (Eroglu et al. 1991). This corresponds to a flash duration of 0.05 millisecond, with a displacement of $0.1 \mathrm{~mm}$ during this exposure time. On the other hand, the displacements of water droplets at near nozzle regions were also of the same order at Weber numbers less than 50. Hence, the lower level of flash duration was chosen to be 0.05 millisecond, which was sufficient to 'freeze' the images of moving liquid jet and droplets. Time integrated images of the sprays were also taken by setting the flash duration to 2.5 millisecond. With reference to the velocities mentioned earlier, the droplets get displaced by $5 \mathrm{~mm}$ within this exposure time of 2.5 millisecond leading to the timeintegrated recording on the photographic film.

The aperture openings in the camera were adjusted individually to suit the light intensity. Complete opening of aperture (f/3.5) was set while taking the slitlight photo and medium opening of $(f / 5)$ to minimum opening of (f/16) was set in the other cases. Two different rating photographic films namely 400 ASA and 1600 ASA were used to capture the images to suit the lighting condition and exposure time.

Different lighting techniques employed in the study are schematically shown in Fig. 4. In the backlight photographic technique (shadowgraph) the flash unit synchronised with the camera shutter was placed behind a translucent glass sheet and camera aperture was directly exposed to the illuminated spray (Fig. 4a). The translucent ground glass sheet diffuses the light from the flash to a wider region thus providing a uniform illumination of spray over the entire cross section.

In case of scattered light photography, the flash lamp of $1 \mathrm{KW}$ was placed at an angle of $120^{\circ}$ from the line of sight of camera and the spray was illuminated (Fig. 4b). 
Care was taken to avoid direct exposure of camera lens to the light from the lamp and only the scattered light from the water droplets in the spray was captured.

The flash unit was mounted on the camera while photographing the external regions of spray (Fig. 4c). In this mode, the illuminating light source and the camera aperture were in the same plane resulting in capturing the backward reflected light from the water droplets.

In the slitlight photography technique, the flashlight from the projector converged to a sheet of light of thickness $1 \mathrm{~mm}$ at the spray centerline and the camera was placed with its axis normal to the sheet of light (Fig. 4d).

\section{RESUlts AND DisCuSSION}

In this section the photographs taken with different techniques are analysed in the first part and in the second part the breakup of liquid jet from an airblast atomizer is dealt with.

\subsection{Photographic Analysis}

The liquid jet issuing out of a nozzle shows an initial region of intact water column wherein the instabilities are set in, which further lead to atomization into liquid lumps and droplets. The photographing then involves the challenge of capturing the images of the intact and fluctuating cylindrical liquid column, comparatively slow moving odd shaped liquid lumps and nearly spherical smaller droplets that are being carried away by the air stream at high velocity. For a pilot comparative study of images of liquid jet and droplets under various lighting conditions, photographs were also taken with a few transparent and opaque objects. These include a round bottom glass flask with a long cylindrical neck, and a brass nozzle. The images of cylindrical water jet are comparable with those of cylindrical neck while droplet images can be compared with the photographs of the spherical bottom of the flask. Since the images of cylindrical neck and spherical bottom of the flask are taken under stationary conditions, the effect of time averaging in case of actual spray images can be studied in comparison with these images. The images of brass nozzle (opaque body) are considered for the sake of completion of discussion of the effect of various lighting conditions used. The images of the opaque body are also useful in the study of the image quality of the spray of a nearly opaque liquid. The photographs of images of round bottom glass flask and the nozzle obtained with different lighting techniques are shown in Fig. 5.

The first photograph (Ia) in Fig. 5 is the image of the empty round bottom flask obtained by shadowgraphy method. The flask has a thick walled neck (thickness $\approx 0.2 \mathrm{~mm}$ ). The image shows a bright zone within each of the sidewall of the neck accompanied by the dark zones on either sides. This can be explained with the help of principle of steering of wavefronts of light in a medium of non-uniform density.

The principle of wave motion of light rests on the fact that the speed of light varies with density of the medium through which it is passing. The speed $c$ in any medium is related to the speed $c_{o}$ in vacuum by the index of refraction $n=c_{o} / c$. For a given substance and for the given wavelength of light, the index of refraction is a function of density $n=n(\rho)$. When light passes through a varying density medium, it experiences retardation in proportion to its density. This has two effects-one is the turning of wavefronts, a property used in the shadowgraph and Schlieren, and the other, the relative phase shift of different rays that is useful in obtaining interferometer images.

A schematic representation of turning of wavefronts due to variation in density (or refractive index) in the medium is shown in Fig. 6. A parrallel beam of light entering a medium in which the density (or refractive index) is increasing in the negative $\mathrm{x}$-direction experiences a deflection towards the same direction (Fig. 6a). This results in bright regions where the rays crowd together and dark regions where they diverge (Fig. 6b). This is evident in the image Ia in Fig. 5 of the empty thick walled neck of the round bottom flask. The normal illumination on the screen corresponds to the unchanged spacing between rays. Thus, the shadow effect depends not on the absolute deflection but on the relative deflection of rays, that is, on the rate at which they converge on emerging from the object (Liepmann and Roshko 1963). Thus, in plane flow, the shadow effect depends on the second derivative of the density.

The same cylindrical long neck when filled with water shows (photo IIa in Fig. 5) a dark shade at the borders and average brightness at all other regions without the bright thin line at the centre line of wall as observed in the previous case. The reason is obviously the lower ratio of refractive indices of water (1.33) and glass (1.5). Picture IIIa in Fig. 5 shows the shadow effect in case of an opaque body in which the dark shade is filled inside the boundary of image and the average illumination at all other regions.

A configuration similar to $120^{\circ}$ scattered light technique has been used earlier by Carvalho and Heitor (1998), in which two spot-lights of $1000 \mathrm{~W}$ each have been placed on two sides of the spray at about $120^{\circ}$ to the plane of camera aperture. The image obtained is analysed using CCD camera for determining the spray characteristics. However, this configuration is referred to, as 'backlighting technique' by the authors and the study does not include any explanation for the noted differences of images of shadowgraphs and scattered light technique. In the present study, the term 'backlighting' refers to the shadowgraphy technique to avoid any confusion. In case of $120^{\circ}$ scattered light photographs, the neck of object appears with a bright outer region and dark inner region (pictures $\mathrm{I} b$ and $\mathrm{II} b$ in Fig. 5) just in contrast with the backlight photography. The total internal reflection at the boundaries of transparent objects results in bright boundaries and average illumination at other parts. On the other hand, the opaque body (picture III b in Fig. 5) shows the bright boundary at the illuminated edge and comparatively darker region in the rest of the field. In this case the total internal refraction does not apply, since the body is impervious to light.

The front illumination lighting technique is a commonly adopted method to capture the external appearance of 
the spray and hence has been used right from the early investigators Dombrowski and Fraser (1954) to the recent Vladimir and Vigor (1998) and Shedd and Pautsch (2005). In this method, the back-scattered signals from the object are captured on the photographic film. The photographs (Fig. 5c) taken with this technique are useful in revealing the external features of the object. However, for want of a sliced view through a transparent object, the sheet of light technique is used. In this case, the light scattered by object at $90^{\circ}$ to the incident light is recorded on the photographic film revealing the inner details of the object along the plane of illumination (Fig. 5d). The image of neck of the empty flask shows two bright regions (picture I d in Fig. 5) each at the respective walls under sheet light incidence at right angle to the camera. This is due to the total internal reflection of incident rays at the interfaces of air and glass. With water filled in the flask, the wall which receives the rays first (left wall) appears bright, while the right wall relatively dull (picture II b in Fig. 5). At the first wall the interface is between air and glass and at the second wall interface is between water and glass leading to varied refractive indices at these sections. Added to this, the obscuring of light in the denser medium (water) leads to the noted difference between brightness of the walls. The opaque body (picture III d in Fig. 5) does not allow any light to pass through. This technique would prove useless in deriving any useful information for opaque liquids.

After having studied the pilot photographs of water filled round bottom flask with the long cylindrical neck, the sprays from the airblast atomizer and swirl atomizer are analysed. The image of the water filled cylindrical glass tube Fig. 5 is comparable with that of intact cylindrical water jet in the initial stages of spray formation (Figs. 7, 8 and 9). Similarly, the shadow image of water filled thin bottom of the flask is comparable with that of a spherical drop. In a shadowgraph of liquid cylindrical jet, hollow cone or water drop, thick shadow and an adjacent relatively thinner shadow at the internal region (Figs.7a, 8a and pictures I in Fig. 9) mark the boundary of liquid mass. With a lower flash duration of 0.05 millisecond, the instant shadow picture of the spray pattern is arrested on the photographic film (pictures I in Figs.7a and 8a and 9). This provides useful information on the inner details of the spray such as the breakup length and breakup mechanism. In the backlighting technique, the smaller droplets may become completely transparent to the backlight leaving no significant signature on the film thereby loosing some important information. This effect is more pronounced in the case of time integrated photograph (picture II in Figs. 7a and 8a) with the flash duration of 2.5 millisecond, in which only the cylindrical liquid core or hollow sheet of liquid is seen and the finely atomized part of the spray appear blurred, due to relative motion of droplets in the time frame.

The details of density variation in the shadowgraphy will be lost if the screen is not closer to the object, and only dark shade fills the entire image as can be seen from the photographs of Carvalho and Heitor (1998). However, in the present study, the camera lens is directly focused to the midplane of the spray, hence the effective location of screen is at the axis of the spray, making it possible to record all the finer details of shadow on the film.

In case of $120^{\circ}$ scattered light photographs, the liquid jet/sheet appears with a bright outer region and the dark inner region (Figs. 7b, 8b and 9 II) just in contrast with the backlight photography (Figs. 7a and 8a and 9I) and as also discussed earlier with respect the images of round-bottomed flask. The total internal reflection at the boundaries of transparent objects results in bright boundary and average illumination at other parts. However in the case of time integrated pictures (pictures II in Figs. 7b, 8b and 9II), the motion of droplets in the timeframe result in 'milky' streaks of droplets. Due to relatively longer exposure time ( 2.5 millisecond) in this case, the streak line followed by even the smallest drop is recorded on the film. This defines the boundary of the spray (Fig. 7b) and the cone angle can be measured with ease.

The images of instant shadowgraphs and $120^{\circ}$ scattered light exhibit entirely different characteristics of the spray (see Fig. 10). The characteristics such as inner details of spray, jet breakup, the surface wave generation and blow-up, and secondary atomization from the droplets formed by primary atomization etc., are clearly evident in the instant shadowgraph technique. On the other hand, the time integrated scattered light image depicts the external characteristics of the spray such as spray width, cone angle and dispersion. The discussions of jet breakup processes as observed from these images are given in the next section.

Comparing the images of instant shadowgraph (pictures I in Fig. 9) and time integrated $120^{\circ}$ scattered light images (pictures II in Fig. 9), the boundary of cylindrical water jet appears thicker and wider in the latter. The same is the case with the spray from the swirl atomizer (pictures $\mathrm{Ib} \& \mathrm{IIb}$ in Fig. 8). As mentioned earlier, the photographs taken with $120^{\circ}$ scattered light in these pictures have longer exposure time resulting in the time-integrated image of oscillations of surface waves on the jet/sheet. The instantaneous images obtained with instant backlight photography (pictures I Fig. 7a \& Fig 8a) clearly arrest these waves resulting in the appropriate scaling of the jet/sheet. Without these oscillations the width of the images in both the cases must appear equal as can be seen in pictures II Figs. $5 \mathrm{a}$ and $5 \mathrm{~b}$ of empty and water filled round bottom flask.

In The-front illumination lighting technique, the backscattered signals from the liquid mass are captured on the photographic film. Due to weak reflective signal of smaller water droplets, the photograph appears dull in the case of spray from an airblast atomizer (Fig. 7c). The liquid sheet from the swirl atomizer shines in these photographs (Fig. 8c) due to comparatively higher signal strength from the large surface area of sheet. This technique fails to reveal any internal details of the jet/sheet breakup as aresult of masking of inner regions by the droplets at the outer regions. However, the external details of spray can be well captured by this method. 
In the case of sheet-light technique, the light scattered by water droplets perpendicular to the incident light is recorded on the photographic film. It may be observed that the images of $120^{\circ}$ scattered light and sheet light technique appear similar (Figs. 7b and 7d) except for the differences noted as under. Since, the intensity of scattered light at $90^{\circ}$ to the incident beam is less than that at $120^{\circ}$, some information on smaller droplets is lost. For this reason, the spray appears shorter and narrow. On the other hand, this technique proves useful in the measurement of the cone angle and sheet breakup position in case of spray from swirl atomizer (Fig. 8d). The photographs of Bazarov and Yang (1998) and Sivakumar and Raghunandan (1996) also favour the use of sheet light technique for the analysis of spray from swirl atomizers. With the camera placed at $90^{\circ}$ to the sheet light source, a combination of powerful light source and image capturing device is essential to get a reasonably bright image. However, with suitable instrumentation this technique can be exploited to yield the tomographical images by capturing the images at different cross sections as demonstrated by Cavalire et al. (2003).

It is thus clear that the information obtainable from each technique is different and that a combination of different techniques may be necessary for proper diagnosis. Table 1 summarises the information obtained by adopting different lighting techniques in the spray field.

It is clear from the table that the instant backlight technique is useful for the study of breakup phenomenon, while the time integrated $120^{\circ}$ scattered light technique is useful in tracking even the smaller droplets in the spray field. Hence, these techniques are adopted in the following section, in which the breakup phenomenon are dealt in detail.

\subsection{Jet Breakup}

In this section the jet breakup mechanism in a coaxial airblast atomizer is reviewed and the observed breakup regimes in the current configuration are discussed.

Considerable attention has been given by the investigators in the field to explore the jet atomization and droplet breakup in still air and in co-flowing airblast environment, and the various regimes of breakup. Many hypotheses have also been floated. A comprehensive summary of liquid jet breakup and secondary atomization is found in the Lefebvre's (1989) work and Carvalho et al. (2002). Jet breakup in coflowing air stream is shown to be a complex phenomenon that is sensitive to many parameters such as geometry of the atomizer, the fluid properties, the ambient conditions and the jet velocity and momentum. The basic wave growth on the jet surface due to turbulence in itself (Mayer 1994) and due to the coflowing air stream (Eroglu and Chigier 1991) leads to sinuous (antisymmetric) and dilational (symmetric) waves that grow and finally leads to the jet disintegration (Mayer 1994). The liquid jet intact length (or breakup length) defined as the length of jet that is hydraulically connected to the nozzle exit (Eroglu et al. 1991) is one of the commonly used parameter in airblast atomization literature. Although different techniques such as electrical resistance, and flow visualization are used for measuring the breakup length, the variation in such measurements is as high as $\pm 15 \%$ (Eroglu et al. 1991).

The available literature considers the atomization as a jet breakup phenomenon in which the thinning of jet due to the growth of surface wave and oscillation of the jet leading to detached liquid mass which further breakup due to aerodynamic interaction with the coflowing air stream. These observations are mainly based on the evidences of shadowgraphy images obtained at different flow rates of fluids. Based on the physical appearance and the internal wave growth mechanism, various modes of disintegration namely, Rayleigh breakup, membrane breakup, fibre breakup and super pulsating fibre breakup have been identified in the process of spray formation in an airblast atomizer (Chigier and Reitz 1995; Farago and Chigier 1991). These regimes can be readily described by the photographic techniques discussed earlier.

In Rayleigh breakup regime (Fig. 9a to d), a combination of sinuous and dilational waves on the jet surface is seen. The wavelengths increase in downstream region and the grown up dilational waves breakup at nodes, while sinuous waves breakup at crests leading to the atomization (Carvalho et al. 2002). The characteristic of Rayleigh breakup is that the maximum diameter of drops produced due to breakup is twice the jet diameter. At higher airflow rates, the jet develops into thin liquid sheet (membrane) and breaks due to Kelvin-Helmholtz instability (Fig. 9e and 9f). In this case the diameter of drops produced is much smaller than the liquid jet. At even high flow rates, fibre disintegration mode is observed in which the fibres are formed and peel off the jet (Fig. 9g). At low liquid Reynolds number $\left(\rho_{w} V D_{W} / \mu_{w}, V=\right.$ exit diameter of water tube, $D_{W}=$ inner diameter of water tube, $\rho_{w}$ and $\mu_{w}$ are the fluid (water) density and viscosity) and high Weber number $\left(\rho_{A} V_{R}^{2} D_{W} / \sigma, V_{R}=\right.$ relative velocity of air to water at the exit of the nozzle, $\rho_{A}=$ density of air, $\sigma$ is the surface tension of the liquid (water)), an extremely high periodic change between the low and high density region in the spray is observed, that is characterised as superpulsating fibre breakup (Fig. 9h). At this stage, the tapering liquid jet core shows oscillations like snake and breaks up periodically into large ligaments as also observed by Mayer (1994). Another highlight of the scattered light photography in identifying the jet boundaries can be seen in Fig. 9. The liquid jet initially of the size of water nozzle diameter (1 $\mathrm{mm}$ ) bulges at high flow rates of air and gets attached to the liquid tube. The recirculation behind the walls of the water nozzle (wall thickness $=1 \mathrm{~mm}$ ) gets intensified at high flow rates of air resulting in the sucking of smaller droplets in the immediate vicinity into the region of recirculation. With this, the size of the intact liquid volume grows to the size comparable to the external diameter of the liquid nozzle as seen in these photographs. A strong recirculation region right at the exit of water nozzle is expected to induce an early onset of jet instability, thus influencing the primary breakup (Sridhara and Raghunandan 1999). 
The observations made in the jet breakup with the help of shadowgraphs in this study agree with the literature. However, closer looks (Fig. 10) at the corresponding $120^{0}$ scattered light images show the possibility of yet another mechanism of jet disintegration. The Rayleigh type breakup shown in Fig. 10a is accompanied by the corresponding $120^{\circ}$ scattered light image in Fig. $10 \mathrm{~b}$. Point $P_{R}$ in Fig. 10a corresponds to the instantaneous position of formation of first droplet from the liquid core as observed in the shadowgraph. On the other hand, long exposure photograph of the scattered light image (Fig. 10b) shows a few smaller droplets being sheared off the liquid jet far upstream from the point $P_{R}$. These smaller droplets might have escaped from being recorded in the shadowgraph due to the limitation of the backlighting technique discussed earlier. The point identified as $\mathrm{P}_{\mathrm{S}}$ in Fig. $10 \mathrm{~b}$ is the origin of a few streak lines (SL) of such droplets detached from the main liquid core. Many such streak lines in Fig. 10b at downstream region mask the bigger droplets at the core as seen in Fig. 10a. The same is the case with Figs. 10c and 10d respectively. This shows the possibility of generation of a few smaller droplets formed by the shear removal from the surface of liquid jet by the coflowing air stream in the airblast atomizer. However, this needs to be confirmed by further investigation with technique such as high speed cinematography of jet breakup phenomenon in the $120^{\circ}$ scattered light configuration.

\section{Conclusions}

A comprehensive review of flow visualization techniques used for the analysis of spray characteristics has been made. Different lighting techniques used in the still photography have been demonstrated and the images obtained are analysed. It is demonstrated that the information obtainable from each technique is different and that a combination of different techniques may be necessary for proper diagnosis. Photographs taken by these methods are shown to be useful for deriving some useful observations such as jet breakup phenomenon, the internal details and the external appearance of the spray. The article also includes the studies on jet breakup phenomena and the possibility of shear formation of smaller droplets from the surface of liquid jet by the coflowing air even at condition where Rayleigh instability is in its incipient stage.

\section{REFERENCES}

Bazarov G Vladimir, Yang Vigor, (1998). "Liquid propellant rocket engine injector dynamics", Journal of Propulsion and Power, Vol. 14, No. 5, pp. 797-806.

Candel, S., Herding, G., Synder, R., Scouflaire, P., Rolon, C., Vingert, L., Habiballah, M., Grisch, F., Pealat, M., Bouchardy, P., Stepowski, D., Cessou, A., Colin, P., (1998). "Experimental investigation of shear coaxial cryogenic jet flames", Journal of Propulsion and Power, Vol. 14, No. 5, pp. 826-834.

Carvalho, I.S., Heitor, M.V., (1998). "Liquid film breakup model of a prefilming airblast nozzle", Experiments in Fluids, Vol. 24, pp. 408-415.
Carvalho, I.S., Heitor, M.V., Santos, D., (2002). "Liquid film disintegration regimes and proposed correlations", International Journal of Multiphase Flow, Vol. 28 pp. 773-789.

Cavaliere, A., Ragucci, R., Noviello, C., (2003). "Bending and break-up of a liquid jet in a high pressure airflow", Experimental Thermal and Fluid Science, Vol. 27, 449-454.

Chigier, N., Reitz, R.D., (1995). "Regimes of jet breakup and breakup mechanisms (Physical Effects)", Progress in Ast and Aernt., Vol. 166, pp. 109-135.

Dombrowski, N., Fraser, R.P., (1954). "Photographic Investigation into the disintegration of liquid sheets", Philosophical Transactions of Royal Society, London, A., Vol. 247, pp. 101-130.

Eroglu, H., Chigier, N., (1991). "Initial drop size and velocity distributions for airblast coaxial atomizers", Journal of Fluids Engineering, Vol. 113, pp. 453-459.

Eroglu, H., Chigier, N., Farago, Z., (1991). "Coaxial atomizer liquid intact lengths", Physics of Fluids, A., Vol. 3, No. 2., pp. 303-308.

Farago, Z., Chigier, N., (1991). "Classification of round liquid jet disintegration in coaxial air streams", I-Class 91, Paper 73, USA.

Fraser, R.P., Dombrowski, N., Routley, J.K., (1963a). "The production of uniform liquid sheets from spinning cups", Chemical Engineering Science, Vol. 18, pp. 315-321.

Fraser, R.P., Dombrowski, N., Routley, J.K., (1963b). "The filming of liquids by spinning cups", Chemical Engineering Science, Vol. 18, pp. 323337.

Fraser, R.P., Dombrowski, N., Routley, J.K., (1963c). "The atomization of a liquid sheet by an impinging air stream", Chemical Engineering Science, Vol. 18, pp. 339- 353.

Hay, K.J., Liu, Z.C., Hanratty, T.J., (1998). “A Backlight technique for particle size measurements in two phase flows", Experiments in Fluids, Vol. 25, pp. 226-232.

Jones, A.R., (1977). "A review of drop size measurement- The application of techniques to dense fuel spray", Progress in Energy Combustion Science, Vol. 3, pp. 225-234.

Katanoda Hiroshi, Matsuoka Takeshi, Kuroda Seiji, Kawakita Jin, Fukanuma Hirotaka, Matsuo Kazuyasu, (2005). "Aerodynamic study on supersonic flows in high-velocity oxy-fuel thermal spray process", Journal of Thermal Science, Vol. 4, No. 2, pp. 126-129. 
Kuffferath, A., Wende B., Leuckel W., (1999). "Influence of liquid conditions on spray characteristics of internal-mixing twin-fluid atomizers", International Journal of Heat and Fluid Flow, Vol. 20, pp. 513-519.

Lefebvre, A.H., (1989). Atomization and sprays, Hemisphere Publishing Corporation, New York.

Eroglu, H., Chigier N., (1991). "Liquid jet instability in coaxial airflow", I-class-91, USA Paper 78, pp. 703-710.

Liepmann, H.W., Roshko, A., (1963). "Elements of gas dynamics",: John Wiley and Sons. pp. 154-167. London.

Mayer, W.O.H., (1994). "Coaxial atomization of a round liquid jet in a high speed gas stream: A phenonomical study", Experiments in Fluids, Vol. 16, pp. 401-410.

Mayer Wolfgang; Tamura Hiroshi, (1996). "Propellant injection in a liquid Oxygen gaseous Hydrogen rocket engine", Journal of Propulsion and Power, Vol. 12, No. 6, pp. 1137-1147.

Rehab, H., Villermaux, E., Hopfinger, E.J., (1997). "Flow regimes of large velocity ratio coaxial jets", Journal Fluid Mechanics, Vol. 345, pp. 357-381.

Shedd T.A., Pautsch, A.G., (2005). "Spray impingement cooling with single and multiple nozzle arrays, Part - II: Visualization and empirical models",
International Journal of Heat and Mass Transfer, Vol. 48, pp. 3176-3184.

Shivakumar, D., Raghunandan, B.N., (1996). "Jet interaction in liquid-liquid coaxial injectors", Journal of Fluids Engineering, Vol. 118 pp. 329334.

Sridhara, S.N., Raghunandan, B.N., (1999). "Studies on the performance of airblast atomizer under varying geometric and flow conditions", AIAA Paper, No. 99-2460.

Soltani, M.R., Ghorbanian, Ashjaee, M., Morad, M., (2005). "Spray Characteristics of a liquid-liquid swirl atomizer at different mass flow rates", Aerospace Science and Technology, Vol. 9 pp. 592-604.

Tikkanen, J., K.A. Gross, C.C. Berndt, V. Pitkaenen, J. Keskinen, S. Raghu, M. Rajala, J. Karthikeyan, (1997). "Characteristics of the liquid flame spray process", Surface and Coatings Technology, Vol. 90, No. 3, pp. 210-16.

Wu, P.K., Hsiang L.P., Faeth G.M., (1995). "Aerodynamic effects on primary and secondary spray breakup", Progress in Ast. and Aernt., Vol. 169, pp. 247-279.

Zheng, Q.P., Jasuja, A.K., Lefebvre, A.H., (1996). "Influence of air and fuel flows on gas turbine sprays at high pressures", The Combustion Institute, $26^{\text {th }}$ Symposium (Intl.) on Combustion.

Table 1 Summary of information obtainable in the spray field from various lighting techniques

\begin{tabular}{|l|l|}
\hline Information obtainable & Lighting Technique \\
\hline $\begin{array}{l}\text { Breakup phenomena, surface wave generation and growth, breakup length } \\
\text { and secondary atomization details. }\end{array}$ & $\begin{array}{l}\text { Instant and time-averaged backlighting } \\
\text { technique }\end{array}$ \\
\hline Spray width, cone angle, penetration and dispersion. & $\begin{array}{l}\text { Instant and time-averaged } 120^{0} \\
\text { scattered lighting technique }\end{array}$ \\
\hline $\begin{array}{l}\text { Cone angle, liquid sheet breakup length and information on inner liquid } \\
\text { sheet in case of coaxial twin fluid swirl atomizer. }\end{array}$ & $\begin{array}{l}\text { Instant and time-averaged sheet } \\
\text { lighting technique }\end{array}$ \\
\hline $\begin{array}{l}\text { External appearance of the spray field, spray width, cone angle, penetration } \\
\text { and dispersion. }\end{array}$ & $\begin{array}{l}\text { Instant and time-averaged front } \\
\text { incident lighting technique }\end{array}$ \\
\hline
\end{tabular}




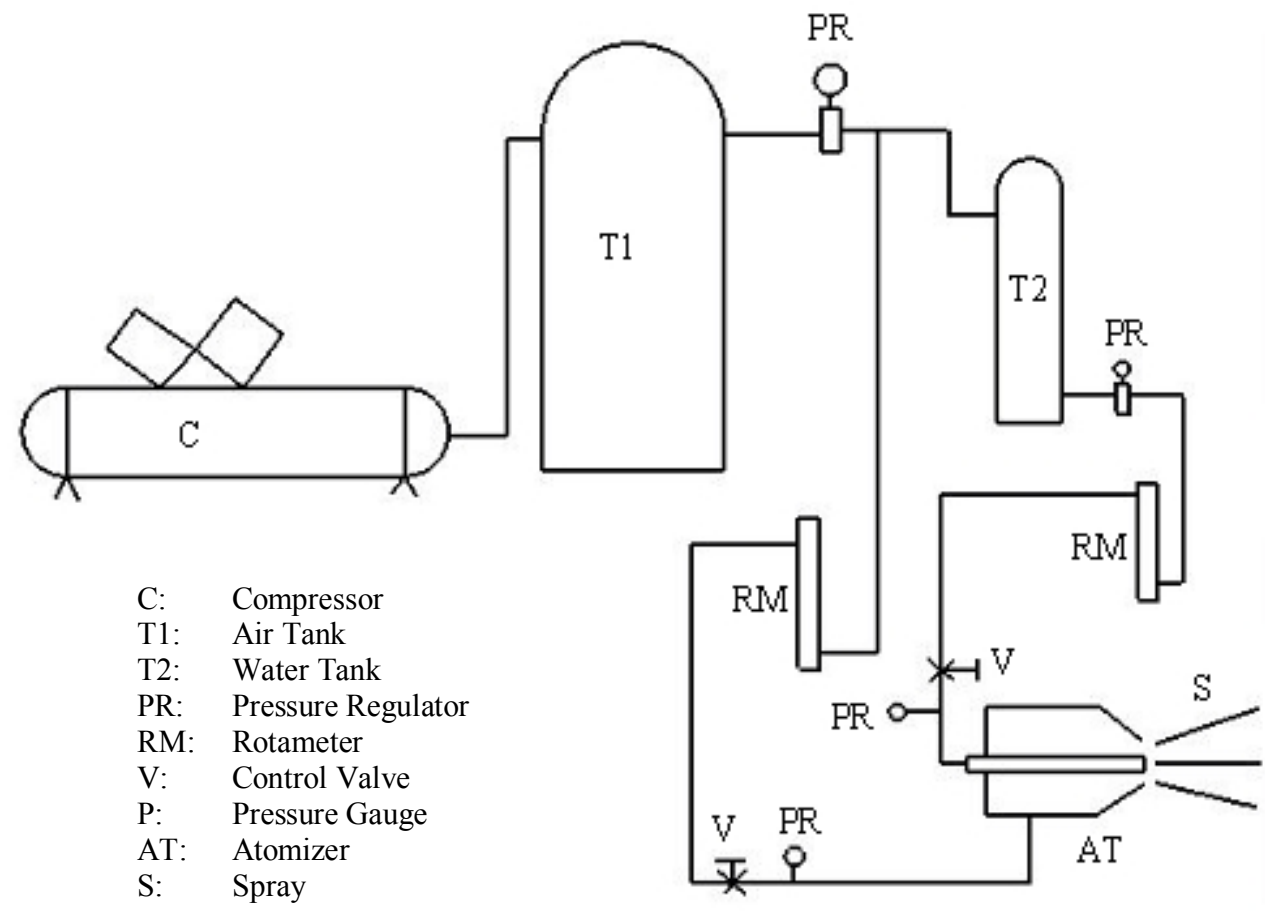

Fig. 3. Schematic of experimental rig

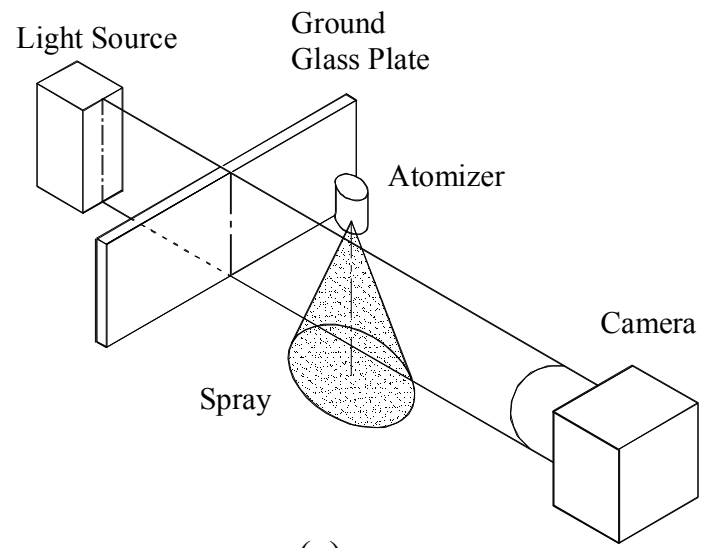

(a)

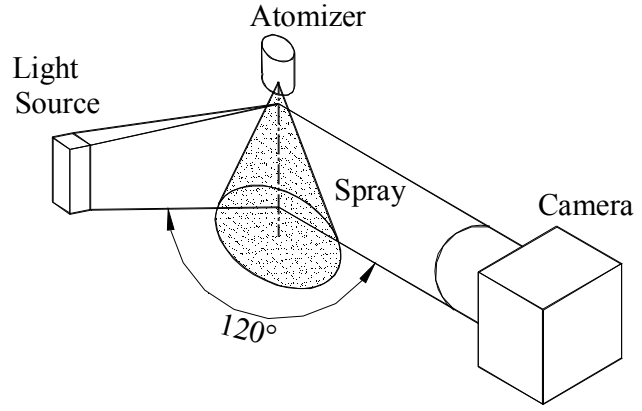

(b)

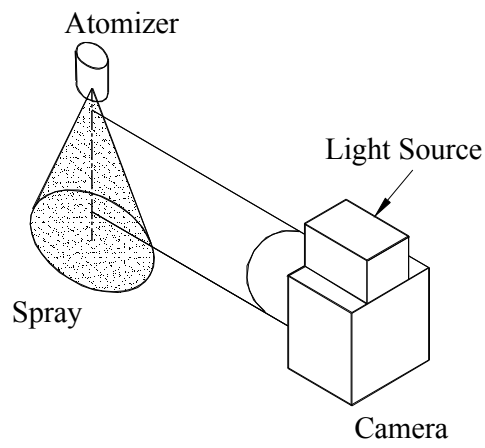

(c)

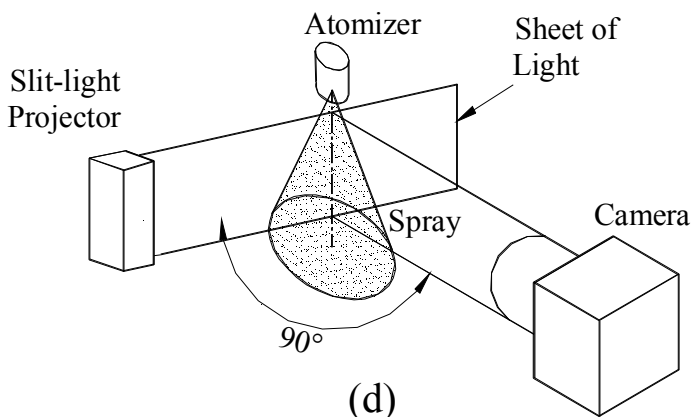

(d)

Fig. 4. Different lightıng tecnniques used in the flow visualization study: (a) backlight with microflash; (b) Halogen lamp at $120^{\circ}$ (scattered light technique); (c) Flash mounted on to the camera (front flash technique); (d) Slit-light from projector. 
(I)

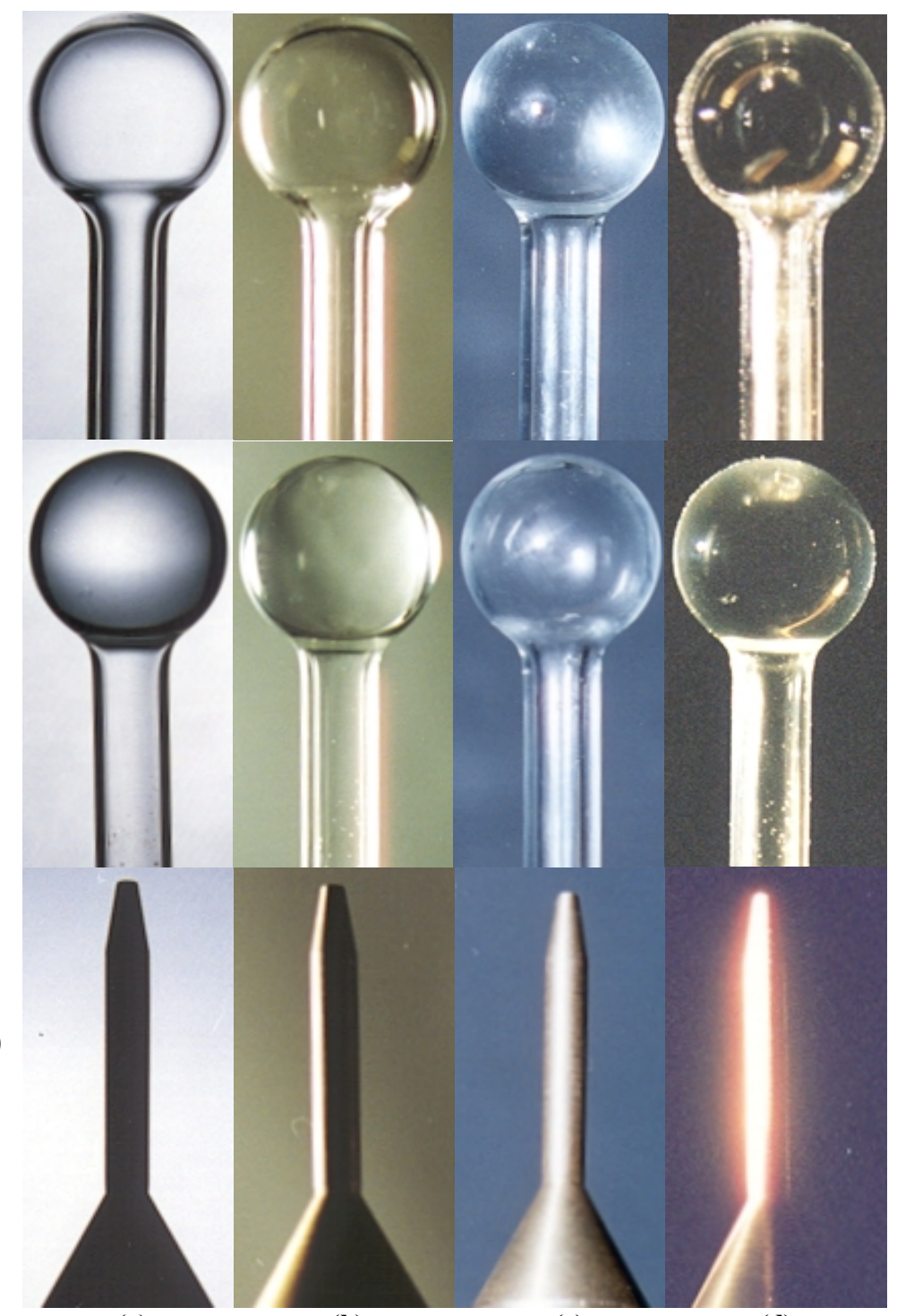

(a)

(b)

(c)

(d)

Fig. 5. Different lighting techniques experimented on different objects: (I) Empty Flask with thin upper bulb and thick bottom neck; (II) Same flask with water filled in it; (III) Opaque brass nozzle tip.

Lighting techniques: (a) backlight technique; (b) $120^{\circ}$ scattered light technique; (c) front incident light technique; (d) sheet-light technique.

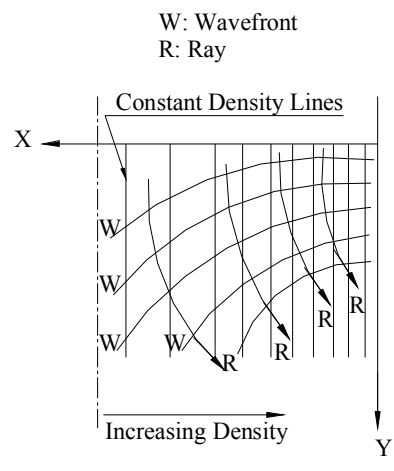

(a)

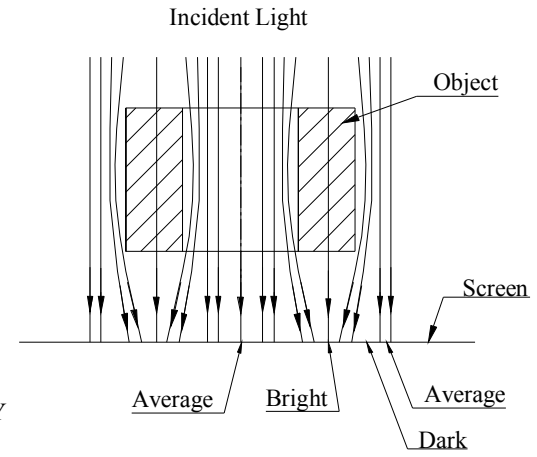

(b)

Fig. 6 Schematic of turning of wavefronts due to difference in density (or refractive index) in the medium. (a) Deflection of wave due to increasing density in negative $x$ direction; (b) The shadow effect with denser medium shown bright and adjacent region dark due to deflection of light in case of transparent medium. 


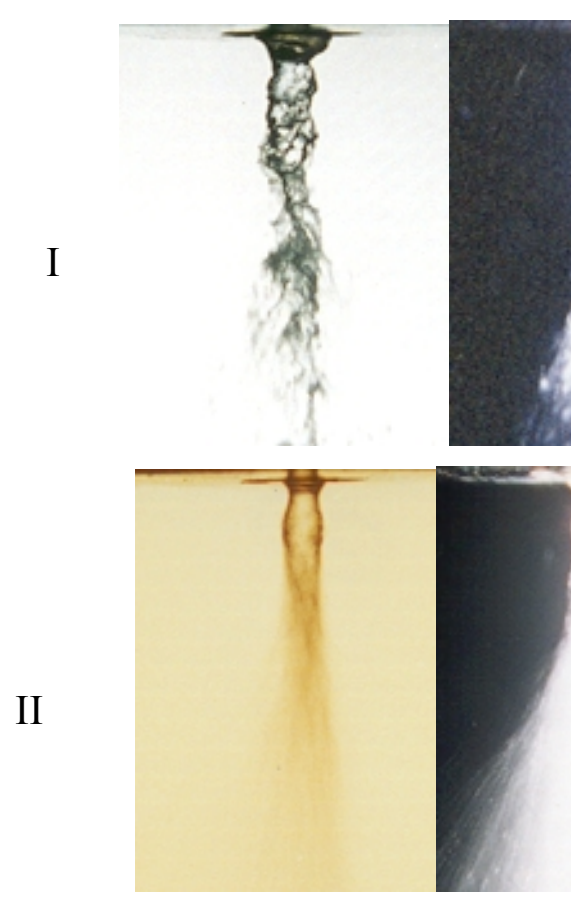

(a)
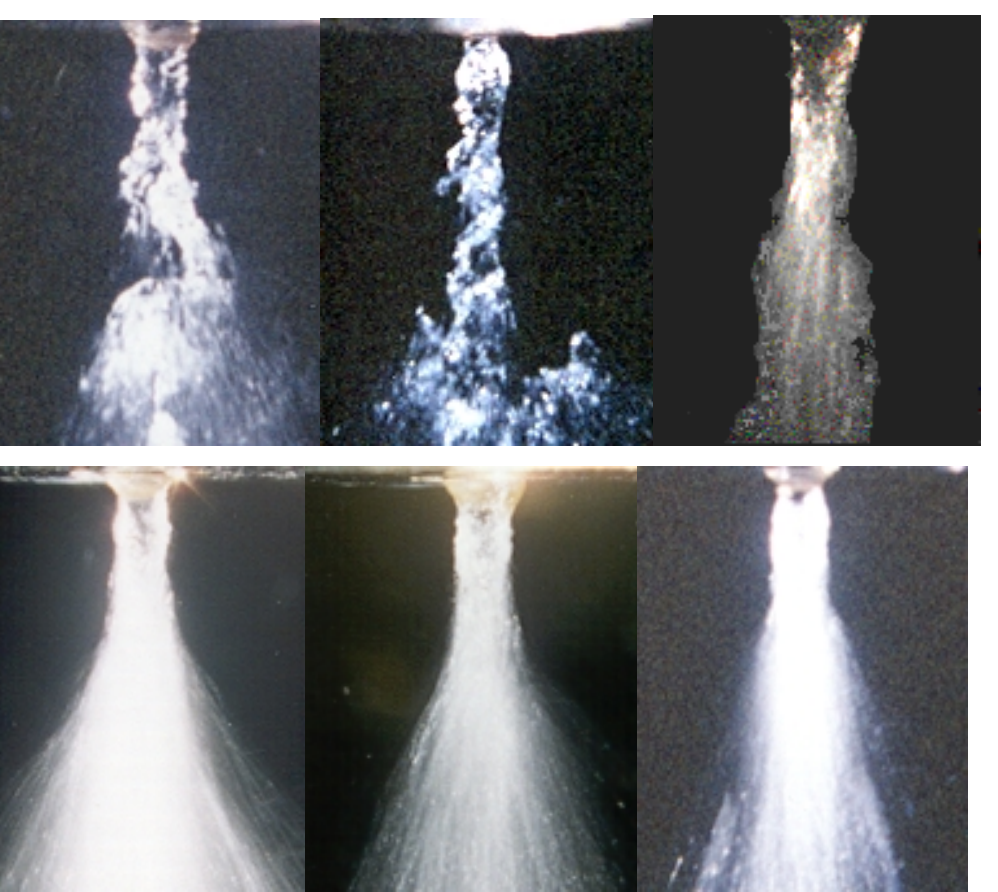

(c)

(d)

Fig. 7. Images of spray from airblast atomizer under varying lighting techniques but under identical flow conditions: (a) backlight technique; (b) $120^{\circ}$ scattered light technique; (c) front incident light technique;

(d) sheet-light technique.

Top row: instant images (flash duration: 0.05 millisecond); Bottom row: time integrated images (flash duration: 2.5 millisecond). Magnification: 1.6:1

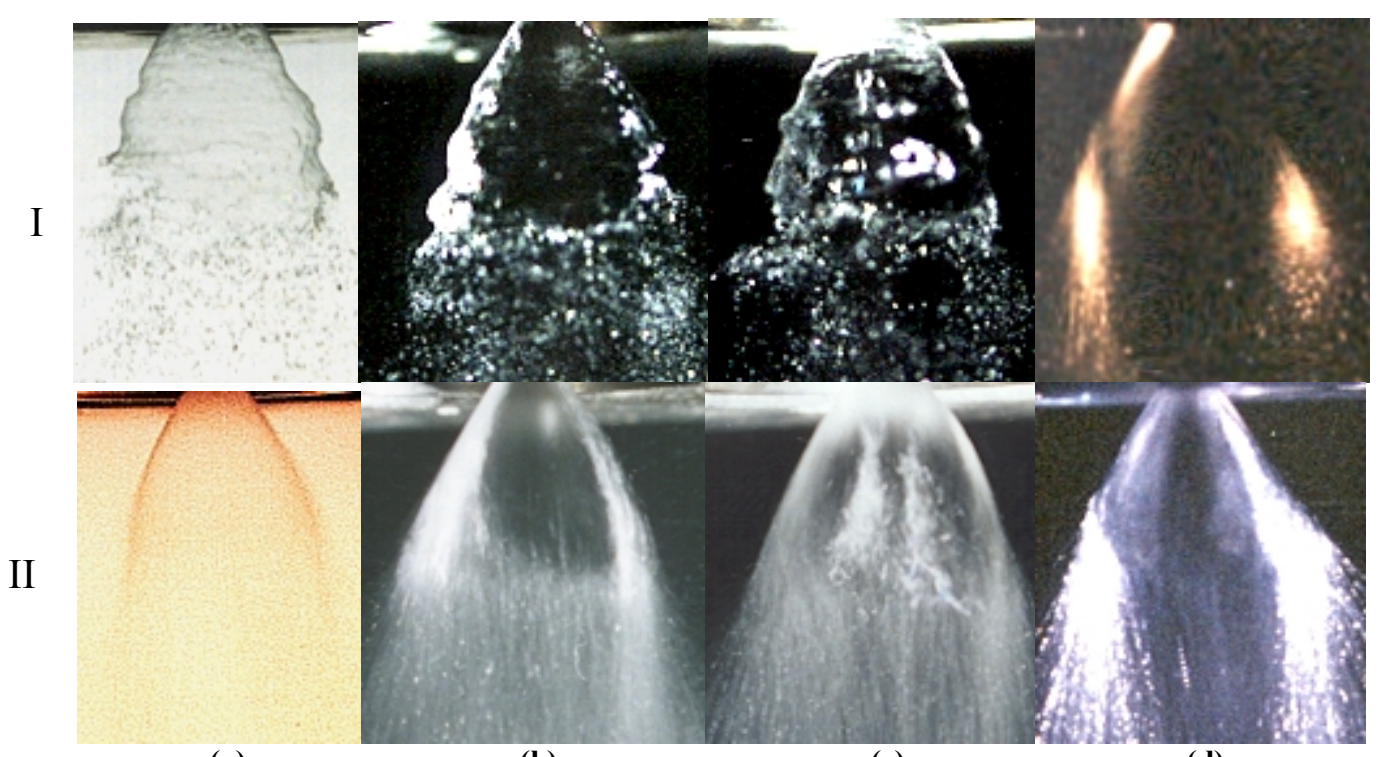

(a)

(b)

(c)

(d)

Fig. 8. Images of spray from swirl atomizer under varying lighting techniques but under identical flow conditions: (a) backlight technique; (b) $120^{\circ}$ scattered light technique; (c) front incident light technique; (d) sheet-light technique.

Top row: instant images (flash duration: 0.05 millisecond); Bottom row: time averaged images (flash duration: 2.5 millisecond). Magnification: 1:4 (reduction). 
S.N. Sridhara and B.N. Raghunandan / JAFM, Vol. 3, No. 2, pp. 111-123, 2010.

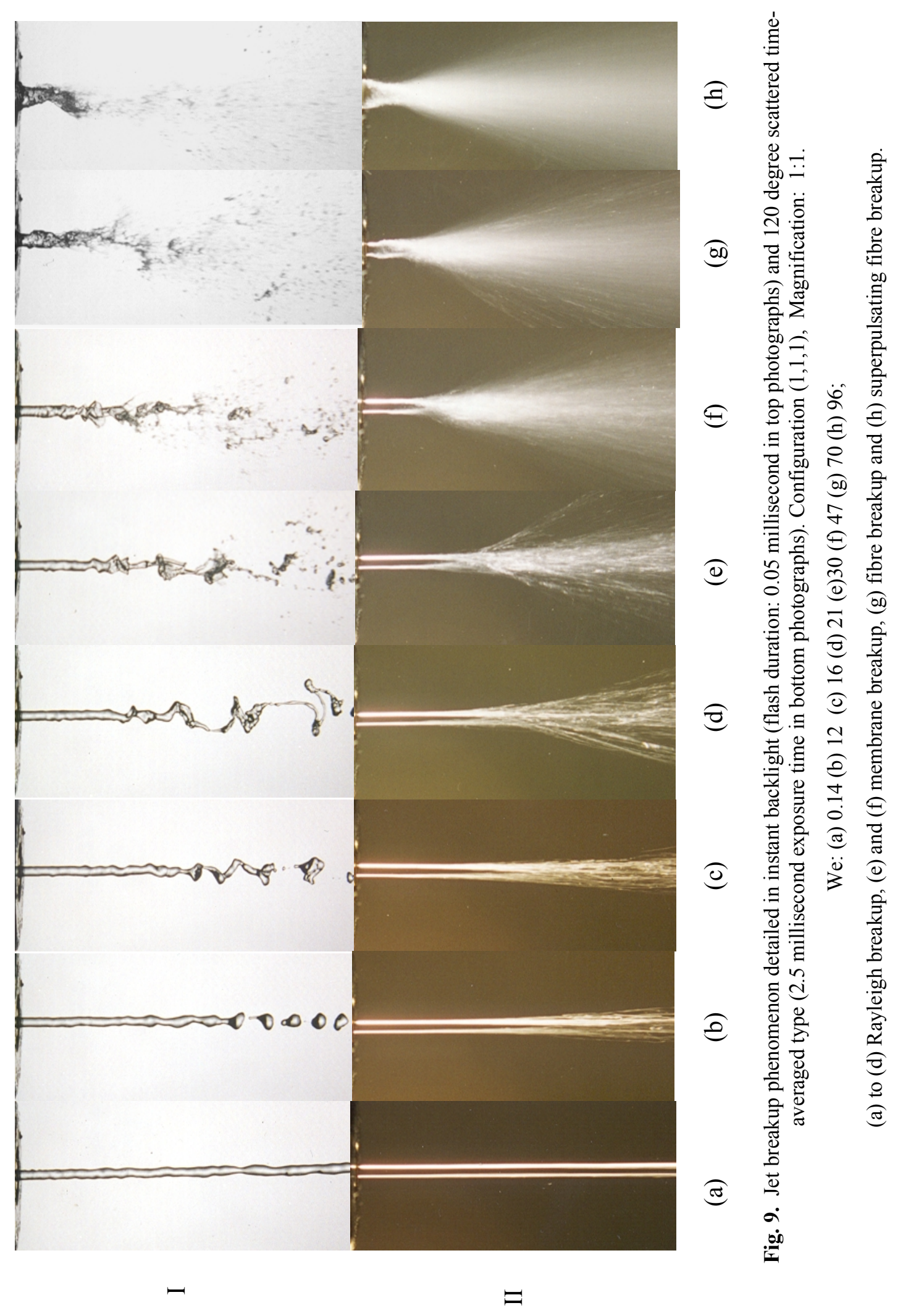




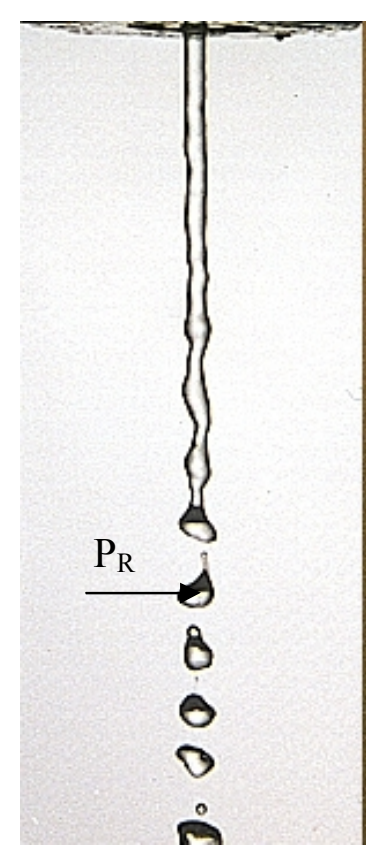

(a)

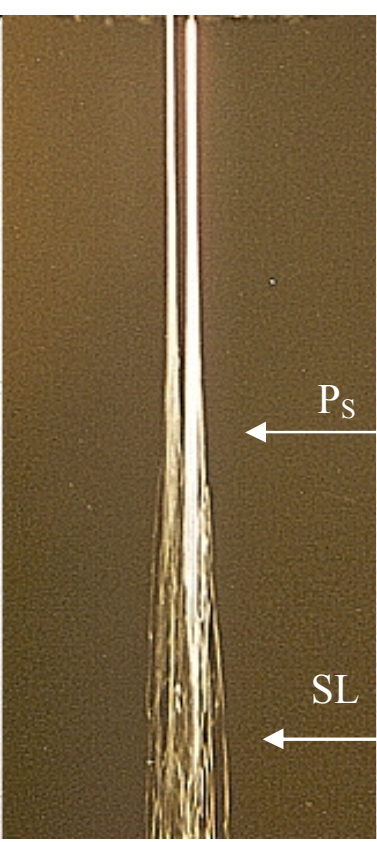

(b)

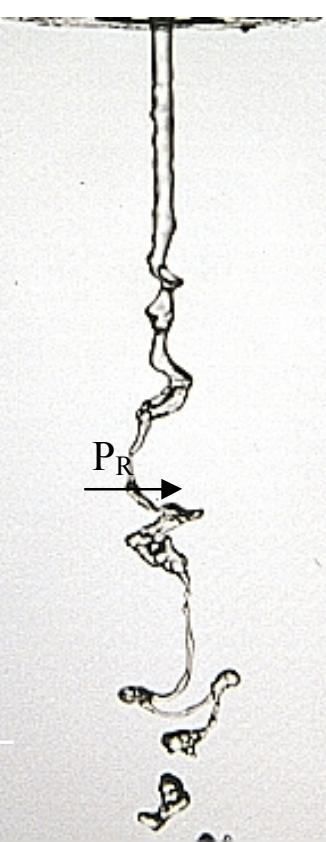

(c)

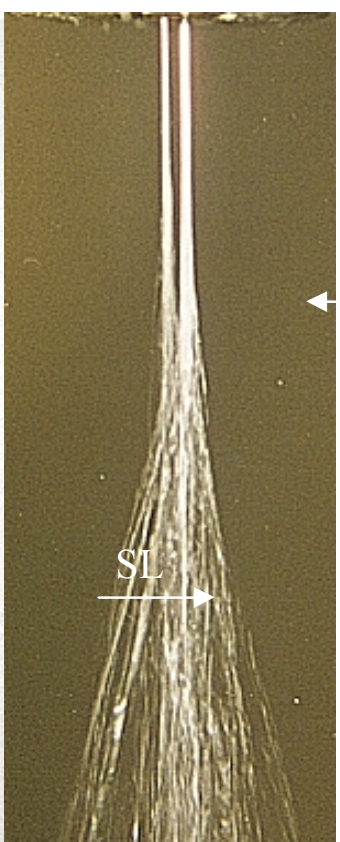

(d)

Fig. 10. Alternative instant backlight and time integrated scattered light photographs of spray from an airblast atomizer. Mass flow rate of water $=2.33 \mathrm{~g} / \mathrm{s}$, Mass flow rate of air $=0.6 \mathrm{~g} / \mathrm{s}$ in (a) and (b) and 0.8 $\mathrm{g} / \mathrm{s}$ in (c) and (d) respectively. Breakup definitions: $\mathrm{P}_{\mathrm{R}}=$ point of initiation of Rayleigh breakup; $\mathrm{PS}=$ point of initiation of droplet formation by shearing of liquid jet surface; $\mathrm{SL}=$ streak line (trace) of one of shear formed droplet. Magnification: 2:1 